http://jmscr.igmpublication.org/home/ ISSN (e)-2347-176x ISSN (p) 2455-0450 crossref DOI: https://dx.doi.org/10.18535/jmscr/v7i8.149

Journal Of Medical Science And Clinical Research

\title{
Hypothyroidism, Maternal Obesity and Self Reported Snoring As Risk Factors for Pregnancy Induced Hypertension
}

\author{
Authors \\ Dr Sahila $\mathrm{M}^{* 1}$, Dr Krisnapriya $\mathrm{SL}^{2}$ \\ ${ }^{1}$ Professor, Dept of Physiology, Govt Medical College, Trivandrum \\ ${ }^{2}$ Junior Resident, Dept of Physiology, Govt Medical College, Trivandrum \\ *Corresponding Author \\ Dr Sahila M \\ Professor, Dept of Physiology, Govt Medical College, Trivandrum, India
}

\begin{abstract}
Background: Pregnancy induced hypertension is one of the most common causes of both maternal and neonatal morbidity affecting about 5-8\% of pregnant women. There are various risk factors for the development of pregnancy induced hypertension. Obesity is increasing at a higher rate in Kerala. Clinical thyroid dysfunction has been associated with pregnancy complications such as hypertension. Snoring is more common in pregnant women compared to non pregnant women. There are only a few studies demonstrating the association of these risk factors with pregnancy induced hypertension in South Indian population.

Objective: To assess the association of hypothyroidism, maternal obesity, self reported snoring as risk factors for pregnancy induced hypertension in patients admitted in antenatal wards in Sree Avittom Thirunal Hospital, Thiruvananthapuram.

Method: This was a case control study involving 50 pregnant women in third trimester diagnosed with pregnancy induced hypertension and 50 normal pregnant women in third trimester without any previous disorders or pregnancy induced complications. Serum TSH and FT4 levels were assessed by chemiluminescentimmuno assay with Beckman Access 2 immunoassay system. Other variables like snoring assessed by providing a questionnaire for snoring to the subjects. BMI assessed by collecting data regarding height and weight from previous visits records. Clinical evaluation involved a proforma based interview with regard to age, parity, history of any other diseases.

Results: Maternal obesity was significantly noted in women diagnosed with pregnancy induced hypertension when compared to normal pregnant women ( $p<0.001$, OR 7.21, CI 2.72-19.1).Pregnant women with history of snoring had significantly higher risk of pregnancy induced hypertension. Obesity and age were independent risk factors of snoring in pregnancy. Elevated TSH levels were found to be significantly associated with pregnancy induced hypertension $(p<0.001)$.

Conclusion: Maternal obesity, self reported snoring and elevated TSH levels (TSH>3mIU/L) can be risk factors for pregnancy induced hypertension.

Keywords: Pregnancy induced hypertension; maternal obesity; snoring; case control study.
\end{abstract}

\section{Introduction}

Hypertensive disorders complicating pregnancy are common and they contribute greatly to maternal and fetal mortality and morbidity worldwide. Hypertension during pregnancy is classified into four categories. 1.Preeclampsia 
Eclampsia 2. Chronic hypertension of any cause 3.Chronic hypertension with superimposed preeclampsia 4.Pregnancy induced hypertension/ Gestational hypertension $^{1}$

Preeclampsia is a pregnancy-specific hypertensive disease with multisystem involvement. It usually occurs after 20 weeks of gestation, most often near term, and can be superimposed on another hypertensive disorder. Preeclampsia, the most common form of high blood pressure that complicates pregnancy, is primarily defined as the occurrence of new-onset hypertension plus newonset proteinuria.

Clinical thyroid dysfunction has been associated with pregnancy complications such as hypertension, preterm birth, low birth weight, placental abruption and fetal death. It has been reported that pregnant women with subclinical hypothyroidism were three times more likely to be complicated by placental abruption.

Obesity is increasing at a higher rate in Kerala. According to National Family Health Survey-3 Kerala ranks second among Indian states next to Punjab for overweight and obesity. During pregnancy many women gain excessive weight which is related to adverse maternal and neonatal outcomes. Prepregnancy maternal adiposity is the strongest modifiable risk factor for hypertensive disorders of pregnancy, ${ }^{2,3}$. The prevalence of obesity was $40.7 \%$ by a cross sectional study conducted in a rural area of Trivandrum district. Another cross sectional study was conducted by Parameshwari et al in 2015 to find out the prevalence of obesity among reproductive age women in rural Kerala and the prevalence of obesity was $47.5 \%$.

Pregnancy is associated with altered breathing during sleep. Snoring is more common in pregnant women compared to non pregnant women. Maternal snoring is a poor prognostic factor for mothers and have greater risk for hypertension and fetal growth retardation. Snoring results from narrowing of upper airway during sleep. Functional residual capacity is decreased by $15-25 \%$ in pregnancy due to increased abdominal mass raising the diaphragm and tracheal shortening are the most likely explanations ${ }^{4,5}$ Also pharyngeal edema and weight gain contribute to upper airway resistance. Nasal congestion and rhinitis are common during pregnancy due to elevated estrogen levels which contribute to upper airway obstruction. High levels of estrogen and progesterone are required to maintain pregnancy. Elevated progesterone levels stimulate central chemo receptors and up regulates ventilatory drive. As a result there is hyperventilation and increased minute ventilation in pregnancy.

Several studies suggested that the prevalence of habitual snoring in woman was strongly dependent on body mass index and neck circumference ${ }^{6}$. The overall prevalence of snoring in South Indian population was $19.5 \%$ according to a study conducted by Balakrishnan et al in Chennai. Studies showing snoring as a risk factor for hypertensive disorders in pregnant women are limited in South Indian population. During the third trimester, total sleep time decreases, while insomnia, nocturnal awakening and day time sleepiness increase ${ }^{7}$. The most common reasons given for third trimester sleep disturbances are fetal movement, backache, general abdominal discomfort, urinary frequency, and heartburn and leg cramps ${ }^{8}$.Upper airway obstruction contribute to increments in blood pressure during sleep.

All these factors pave the way for significance of the study in South Indian population. The aim of the study is to determine whether maternal obesity, hypothyroidism and snoring are risk factors for development of gestational hypertension so that effective measures can be taken to control these factors and prevent the development of hypertension thereby adverse maternal and fetal outcomes

\section{Aims and Objectives}

To assess the association of hypothyroidism, maternal obesity, self reported snoring as risk factors for pregnancy induced hypertension in patients admitted in antenatal ward in SAT Hospital, Thiruvananthapuram. 
Materials and Methods

Study Design

Hospital based Case Control Study

\section{Study Setting}

Sree Avittom Thirunal Hospital, Department of Obstetrics and Gynaecology, Thiruvananthapuram

\section{Study Population}

Pregnant women admitted in Antenatal ward in SAT Hospital, Thiruvananthapuram

\section{Study Subjects}

Cases- All pregnant women in third trimester diagnosed with hypertension who are admitted in antenatal ward in SAT Hospital, Thiruvananthapuram.

Controls- An equal number of gestational age matched pregnant women without any previous disorders or pregnancy induced complications will form controls.

\section{Inclusion Criteria}

Pregnant women in third trimester diagnosed with hypertension (2 elevated blood pressure measurements of $>140 / 90 \mathrm{~mm} \mathrm{hg}$ at least $6 \mathrm{hrs}$ apart without proteinuria) non molar, non multiple gestation form the cases.

An equal number of gestational age matched pregnant women without any previous disorders or pregnancy induced complications will form controls

\section{Exclusion Criteria}

Those who are not willing to give consent.

Those who are having hypertension prior to pregnancy, cardiac, pulmonary and neuromuscular diseases, molar pregnancy, multiple gestation.

Those with history of renal disease and those who are on drugs which alter thyroid function

The development of hypertension anytime during antenatal followup excluded them from control group

\section{Sample Size}

Sample Size is calculated from previous studies134,140,161 based on the formula $n=2 p q$

$(\mathrm{Z} \alpha+\mathrm{Z} \beta) 2$

( P1-p0)2

$\mathrm{P} 0=$ estimated exposure rate among controls. $Z \alpha-$ 0.84 Zß-1.96 R-Odds Ratio
Obtained as 32 cases and 32 controls. But for the present study 50 cases and 50 controls are taken.

Duration of the study-1 year

Data Collection Tool

Questionnaire for snoring (EPWORTH

SLEEPINESS SCALE) used for obtaining data regarding snoring

\section{Estimation of BMI}

Data regarding weight, height obtained from previous visits records.

BMI=weight $(\mathrm{kg}) /($ height in meter)2

Obesity- defined as abnormal or excessive fat accumulation that presents a risk to health. A person with a BMI of 30 or more is considered as obese

For Asian population-Normal BMI-18.5 to 22.9, Underweight $<18.5$, Overweight 23 to 24.9, Preobese 25 to 29.9 , Obese $>30163$

\section{Data Collection Technique}

Cases and Controls are identified from Antenatal ward of SAT Hospital, Thiruvananthapuram. Data regarding height and weight are collected from previous medical records. For calculating BMI, weight on first trimester antenatal visit was taken. A questionnaire for snoring was given to both groups for collecting data regarding snoring. Women may be accompanied by their partners or bystanders during completion of the questionnaire. Blood samples $(4 \mathrm{ml})$ are collected for doing TSH and T4 estimation under aseptic precautions by the principal investigator.

\section{Data Analysis}

Data entered into excel sheet. Quantitative variables were expressed as mean, median, standard deviation and interquartile range. Qualitative variables were expressed as proportion. Comparison of quantitative variables between two groups were analyzed by unpaired $t$ test. Comparison of qualitative variables between the groups were analyzed by Chi square test. Variables which were found significantly associated with hypertension during univariate analysis were subjected to multivariate analysis of binary logistic regression. A $p$ value of $<0.05$ was 
considered statistically significant. SPSS version 16 is used for statistical analysis

\section{Ethical Considerations}

Institutional ethical committee clearance to conduct the study was obtained before starting the study (IEC.No.07/29/2016/MCT). Informed written consent was obtained from the participants. Confidentiality was ensured and maintained throughout the study. No expenses were incurred on the part of the participants.

\section{Observation and Results}

With a view to find out whether hypothyroidism, maternal obesity and self reported snoring are risk factors for pregnancy induced hypertension, a total of 100 pregnant women in third trimester admitted in the antenatal ward of Sree Avittom Thirunal Hospital, Thiruvananthapuram were studied. 50 pregnant women in third trimester diagnosed with pregnancy induced hypertension were taken as the cases. An equal number of gestational age matched pregnant women without any previous disorders or pregnancy induced complications were taken as controls.

Cases and Controls were identified from Antenatal ward of SAT Hospital; Thiruvananthapuram. Data regarding height and weight are collected from previous medical records. For calculating BMI, weight on first trimester antenatal visit was taken. A questionnaire for snoring was given to both groups for collecting data regarding snoring. Women may be accompanied by their partners or bystanders during completion of the questionnaire. The clinical evaluation involved a proforma based interview with regard to age, parity, history of any other diseases. Blood samples $(4 \mathrm{ml})$ are collected for doing TSH and T4 estimation under aseptic precautions by the principal investigator.

\section{Age}

The mean age of the study group was 26 years with a standard deviation of 3.18 .
The mean age of pregnant women in the case group (with $\mathrm{PIH}$ ) was $26.1(\mathrm{SD}=3.18)$ and the mean age of women in control group (without $\mathrm{PIH})$ was $24.3(\mathrm{SD}=3.56)$.

Out of the 50 cases $28 \%$ were in $\leq 23$ age group, $28 \%$ were in $24-26$ age groups, $44 \%$ were in $27+$ age group. Out of the 50 controls $48 \%$ were in $\leq 23$ age group, $26 \%$ were in $24-26$ age group and $26 \%$ were in $27+$ age group.

\section{Body Mass Index}

The body mass index was calculated from height and weight measurement. The mean body mass index of case group was $28.27 \mathrm{~kg} / \mathrm{m} 2(\mathrm{SD}=4.29)$ and the control group was $22.85 \mathrm{~kg} / \mathrm{m} 2(\mathrm{SD}=4.24)$. The difference was statistically significant by independent sample $\mathrm{t}$ test.

Out of the 50 cases $4 \%$ were underweight, $10 \%$ were normal, $8 \%$ were overweight, $30 \%$ were pre Obese, $48 \%$ were obese.

Out of the 50 controls $18 \%$ were underweight, $36 \%$ were normal, $10 \%$ were overweight, $28 \%$ were preobese and $8 \%$ were obese.

\section{Serum TSH Levels}

The mean TSH level of the case group was $2.25 \mathrm{mIU} / \mathrm{L}(\mathrm{SD}=0.90)$ and that of the control group was $1.66 \mathrm{mIU} / \mathrm{L}(\mathrm{SD}=0.70)$. The difference was statistically significant by independent $t$ test between the groups.

Out of the 50 cases none of the individuals had TSH levels $<0.3 \mathrm{mIU} / \mathrm{L}, 76 \%$ had TSH levels of 0 . 3-2.9 mIU/L and 24\% had TSH levels $>3 \mathrm{mIU} / \mathrm{L}$.

Out of the 50 controls $2 \%$ individuals had TSH levels <3mIU/L, 94\% had TSH levels ranging between 0.3 and $2.9 \mathrm{mIU} / \mathrm{L}$ and $4 \%$ had $\mathrm{TSH}$ levels $>3 \mathrm{mIU} / \mathrm{L}$.

\section{Serum FT4 Levels}

The mean FT4 levels in case group was 0.80 with a SD of 0.24 and the mean FT4 levels in control group was 0.90 with a SD of 0.25 .The difference was not statistically significant by independent $t$ test between the groups.

Out of the cases $16 \%$ of the women had FT4 values $<0.6 \mathrm{ng} / \mathrm{dl}$ and $84 \%$ had FT4 values ranging between 0.6 and $1.8 \mathrm{ng} / \mathrm{dl} .8 \%$ of the control group 
had FT4 values $<0.6 \mathrm{ng} / \mathrm{dl}$ and $92 \%$ had values ranging between 0.6 and $1.8 \mathrm{ng} / \mathrm{dl}$.

\section{Gestational Age}

The mean gestational age in case group was 32 weeks $(\mathrm{SD}=1.94)$ and the mean gestational age in the control group was 32 weeks $(\mathrm{SD}=2.10)$. The difference was not statistically significant by independent $t$ test between the groups.

\section{Parity}

Out of the 50 cases $52 \%$ were primigravida and $48 \%$ were multigravida. Out of the 50 controls $60 \%$ were primigravida and $40 \%$ were multigravida.

Out of the 50 cases $52 \%$ were primigravida and $48 \%$ were multigravida. Out of the 50 controls $60 \%$ were primigravida and $40 \%$ were multigravida.

\section{Snoring Frequency among Study Group}

\begin{tabular}{|l|c|c|c|c|c|c|}
\hline \multirow{2}{*}{ Snoring } & \multicolumn{2}{|c|}{ Case } & \multicolumn{2}{c|}{ Control } & \multicolumn{2}{c|}{ Total } \\
\cline { 2 - 7 } & $\mathbf{n}$ & $\%$ & $\mathbf{n}$ & $\%$ & $\mathbf{n}$ & $\%$ \\
\hline Yes & 41 & 82.0 & 16 & 32.0 & 57 & 57.0 \\
\hline No & 9 & 18.0 & 34 & 68.0 & 43 & 43.0 \\
\hline Total & 50 & 100.0 & 50 & 100.0 & 100 & 100.0 \\
\hline
\end{tabular}

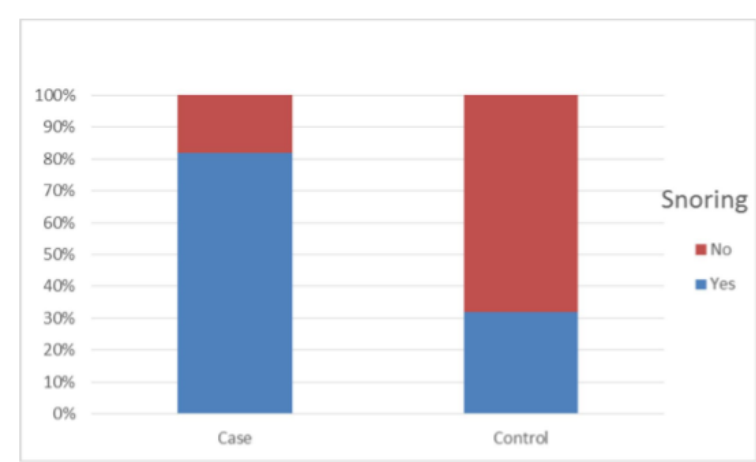

Figure showing percentage distribution of snorers and non snorers among the study subjects

There was significant statistical association between snoring among study subjects and pregnancy induced hypertension ( $\mathrm{p}$ value $<0.001$ ). Out of the 50 cases $82 \%$ had snoring and $18 \%$ didn't snore. Out of the 50 controls $57 \%$ snored while $43 \%$ had no snoring

\section{Risk Factors for snoring in pregnancy in multivariate analysis}

\begin{tabular}{|c|c|c|c|c|}
\hline \multirow{2}{*}{ Variables } & \multirow{2}{*}{$\mathbf{p}$} & \multirow{2}{*}{ OR } & \multicolumn{2}{|c|}{$95 \%$ C.I } \\
\cline { 3 - 4 } & & & Lower & Upper \\
\hline BMI $<30$ & .049 & 1.000 & \multirow{2}{*}{0.997} & 4.538 \\
\hline$>30$ & & 2.127 & & \\
\hline Age $<26$ & .024 & 1.000 & \multirow{2}{*}{1.095} & \multirow{2}{*}{3.756} \\
\hline$>26$ & & 2.028 & & \\
\hline
\end{tabular}

The results indicate that age $(\mathrm{p}=0.024)$ and obesity $(\mathrm{p}=0.049)$ are significantly associated with snoring in pregnancy. 
Logistic Regression Analysis

Multiple Logistic Regression Analysis of Various Parameters with pregnancy induced Hypertension status

\begin{tabular}{|c|c|c|c|c|c|c|c|c|c|c|c|c|c|}
\hline & & \multicolumn{2}{|c|}{ Case } & \multicolumn{2}{|c|}{ Control } & \multicolumn{2}{|c|}{ Total } & \multirow{2}{*}{$\chi^{2}$} & \multirow{2}{*}{ df } & \multirow{2}{*}{$\mathbf{p}$} & \multirow{2}{*}{ OR } & \multicolumn{2}{|c|}{$95 \%$ CI for OR } \\
\hline & & $\mathbf{n}$ & $\%$ & $\mathbf{n}$ & $\%$ & $\mathbf{n}$ & $\%$ & & & & & Lower & Upper \\
\hline \multirow[t]{2}{*}{ Snoring } & Yes & 41 & 82 & 16 & 32 & 57 & 57 & 25.5 & 1 & $<0.001$ & 9.68 & 3.8 & 24.7 \\
\hline & No & 9 & 18 & 34 & 68 & 43 & 43 & & & & & & \\
\hline \multirow[t]{2}{*}{ BMI } & $>30$ & 43 & 86 & 23 & 46 & 66 & 66 & 17.8 & 1 & $<0.001$ & 7.21 & 2.72 & 19.1 \\
\hline & $<30$ & 7 & 14 & 27 & 54 & 34 & 34 & & & & & & \\
\hline \multirow[t]{2}{*}{ TSH } & $>3.0$ & 12 & 24 & 2 & 4 & 14 & 14 & 8.31 & 1 & 0.004 & 7.58 & 1.6 & 35.9 \\
\hline & $<3.0$ & 38 & 76 & 48 & 96 & 86 & 86 & & & & & & \\
\hline \multirow[t]{2}{*}{ Age } & $>26$ & 22 & 44 & 13 & 26 & 35 & 35 & 3.56 & 1 & 0.059 & 2.24 & 0.96 & 5.2 \\
\hline & $<26$ & 28 & 56 & 37 & 74 & 65 & 65 & & & & & & \\
\hline
\end{tabular}

\section{Binary Logistic regression}

\begin{tabular}{|c|c|c|c|c|}
\hline \multirow[t]{2}{*}{ variables } & \multirow[t]{2}{*}{$\mathbf{p}$} & \multirow[t]{2}{*}{ OR } & \multicolumn{2}{|c|}{$\begin{array}{c}\text { 95\% C.I.for } \\
\text { EXP(B) }\end{array}$} \\
\hline & & & Lower & Upper \\
\hline $\mathrm{BMI}>30$ & .033 & 3.392 & 1.105 & 10.412 \\
\hline $\mathrm{TSH}>3.0$ & .173 & 3.244 & .597 & 17.631 \\
\hline Age $>26$ & .103 & 2.372 & .841 & 6.689 \\
\hline Snoring & .001 & 6.058 & 2.179 & 16.845 \\
\hline
\end{tabular}

On multiple logistic regression analysis, maternal obesity $\left(\mathrm{BMI}>30 \mathrm{~kg} / \mathrm{m}^{2}\right)$, high TSH levels $(>3.0 \mathrm{mIU} / \mathrm{L})$ and self reported snoring were independently associated with Pregnancy induced hypertension. On binary logistic regression $\mathrm{BMI}>30 \mathrm{~kg} / \mathrm{m}^{2} \quad(\mathrm{p}=0.03)$ and snoring $(\mathrm{p}=0.001)$ was associated with pregnancy induced hypertension.

\section{Discussion}

Hypothyroidism in pregnancy induced hypertension

Hypertensive disorders of pregnancy are a leading cause of maternal and perinatal mortality and morbidity in India and worldwide. Hypothyroidism is one of the major risk factors for the development of pregnancy induced hypertension. Normal pregnancy is associated with mild hyperthyroxinemia due to the effect of high levels of oestrogen ${ }^{9,10}$.

In the present study serum TSH levels were significantly increased in women diagnosed with pregnancy induced hypertension than in normal pregnant women with a $\mathrm{p}$ value of $<0.001$. The mean TSH levels in women having PIH was $2.25 \pm 0.9 \mathrm{mIU} / \mathrm{L}$ and that of normal pregnant women was $1.66 \pm 0.70 \mathrm{mIU} / \mathrm{L}$. This was in 
agreement with Divya Sardana et al who reported high TSH levels in women with PIH as compared to normotensive pregnant women. Kumar and coworkers in 2005 reported higher incidence of biochemical hypothyroidism in preeclamptic women as compared to control subjects ${ }^{11}$.Larijani et al in 2004 also reported that serum levels of TSH were higher in women with preeclampsia compared with normal pregnancy which is in agreement with the present study ${ }^{12}$.

Hypothyroidism has been listed as one of the causes of high blood pressure. Maternal thyroid hormone excess or deficiency can influence maternal and fetal outcome at all stages of pregnancy and can interfere with ovulation and fertility $^{13,14}$. Wolfberg AJ found that women with hypothyroid disease were more likely to havechronic hypertension $(2.3 \%$ vs $1.2 \%, \mathrm{p}=0.03)$ and had an increased risk of preeclampsia $(4.3 \%$ vs $2.6 \%, \mathrm{p}=0.03$ ) as compared to women without thyroid disease. Mild alteration in the thyroid hormones might occur due to non thyroidal illness acting as a stress factor. Increase in TSH levels in maternal serum correlated with the severity of PIH and high levels of endothelin. The endothelial dysfunction plays an important role in the pathogenesis of PIH. Nitric oxide, a vasodilator released from the endothelial cells regulates secretion of thyroid hormones by modulating regional blood flow in an animal study ${ }^{15}$.

In the present study serum FT4 levels were also compared between diagnosed PIH subjects and normal pregnant women and the difference was not statistically significant between the groups. The mean FT4 levels in the case group was 0.80 with SD of 0.24 and the mean FT4 levels in the control group was 0.90 with a SD of 0.25.The $\mathrm{p}$ value was $0.064 .82 \%$ of the PIH subjects and $92 \%$ of the normal pregnant women had a normal range of FT4 levels. This was in accordance with Qublan et al who performed a study on 27 preeclampsia cases and reported no significant differences in the levels of FT4 between preeclampsia cases and normal pregnant group.
The present study results show that there is a significant increase in the levels of TSH in pregnant women diagnosed with $\mathrm{PIH}$ compared to normal pregnant women. FT4 levels were in normal range for majority of the PIH subjects. So it can be concluded that women diagnosed with $\mathrm{PIH}$ are suffering from subclinical hypothyroidism.

Maternal obesity and pregnancy induced hypertension

Obesity is becoming an epidemic worldwide. Maternal obesity confers an increased risk of hypertensive disorders of pregnancy and a leading cause of maternal and fetal morbidity and mortality.

In the present study the mean body mass index of the PIH subjects was $28.27 \mathrm{~kg} / \mathrm{m} 2(\mathrm{SD}=4.29)$ and that of the normal pregnant women was $22.85 \mathrm{~kg} / \mathrm{m} 2(\mathrm{SD}=4.24)$ and the difference was statistically significant by independent $t$ test with a $\mathrm{p}$ value of $<0.001$. Out of the $50 \mathrm{PIH}$ subjects $4 \%$ were underweight, $10 \%$ were normal, $8 \%$ were overweight, $30 \%$ were preobese, $48 \%$ were obese. Out of the 50 controls $18 \%$ were underweight, $36 \%$ were normal, $10 \%$ were overweight, $28 \%$ were preobese and $8 \%$ were obese. This was in agreement with Corrie Macdonald-Wallis et al who found out that greater prepregnancy weight and gestational weight gain in early pregnancy were independently associated with an increased risk of gestational hypertension. Crane JM et al and Fortner et al also reported a positive relationship of excess gestational weight gain with a risk of hypertensive disorders of pregnancy Mandal et al in 2011 reported that obese pregnant women were at increased risk of pregnancy induced hypertension (12.32 vs. 2.36\%) in comparison with average weight pregnant women $^{16}$. A case control study conducted in Shahid Akbarabadi Hospital of Obstetrics and gynecology in Tehran found that prepregnancy BMI more than $30 \mathrm{~kg} / \mathrm{m} 2$ had 4.5 fold risk of developing gestational hypertension ${ }^{17}$. Subjects with gestational weight gain of more than the recommended value had an approximately 3 fold 
risk of gestational hypertension compared to those who had normal gestational weight gain. The present study findings are consistent with the above mentioned results.

Snoring and Gestational hypertension

High levels of estrogen and progesterone are required to maintain pregnancy. Along with estrogen and progesterone, the changes occurring during pregnancy in prostaglandin and cortisol levels also have an impact on the respiratory physiology. Elevated progesterone levels stimulate central chemo receptors and up regulates ventilatory drive. As a result there is hyperventilation, increased minute ventilation during pregnancy. Snoring is one of the major risk factors for pregnancy induced hypertension. Sleep disordered breathing is a spectrum of respiratory abnormalities due to the partial or complete collapse of the upper airway during sleep leading to intermittent hypoxia and fragmentation of sleep. These disorders are commonly seen in pregnant women, particularly in the third trimester.

In the present study there was a significant statistical association between snoring and pregnancy induced hypertension with a $\mathrm{p}$ value of $<0.001$. Out of the 50 subjects with PIH $82 \%$ had snoring and $18 \%$ didn't snore. Out of the 50 controls $57 \%$ snored while $43 \%$ had no snoring. This was in accordance with Izci et al who reported regular snoring in $85 \%$ of the pregnant population with gestational hypertension. Gislason et al also demonstrated a strong correlation between hypertension and snoring in pregnancy. Franklin et al reported that $23 \%$ of the preeclamptic women snored.

\section{Conclusion}

1) Maternal obesity was significantly associated with pregnancy induced hypertension

2) Self reported snoring which is an indicator of sleep disordered breathing can be a risk factor for pregnancy induced hypertension.
3) TSH levels were found to be significantly higher in women diagnosed with pregnancy induced hypertension.

4) Serum FT4 levels were in normal range in women diagnosed with pregnancy induced hypertension.

5) Age and obesity can be independent risk factors for snoring in pregnancy.

\section{References}

1. Brown MA, Lindheimer MD, Swiet M de, Assche AV, Moutquin J-M. The Classification and Diagnosis of the Hypertensive Disorders of Pregnancy: Statement from the International Society for the Study of Hypertension in Pregnancy (ISSHP). Hypertension in Pregnancy. 2001 Jan 1;20(1):ix-xiv

2. Thadhani R, Stampfer MJ, Hunter DJ, Manson JE, Solomon CG, Curhan GC. High body mass index and hypercholesterolemia: risk of hypertensive disorders of pregnancy. Obstet Gynecol. 1999 Oct;94(4):543-50.

3. O’Brien TE, Ray JG, Chan W-S. Maternal body mass index and the risk of preeclampsia: a systematic overview. Epidemiology. 2003 May;14(3):368-74.

4. Craig DB, Toole MA. Airway closure in pregnancy. Can AnaesthSoc J. 1975 Nov;22(6):665-72.

5. Gee JB, Packer BS, Millen JE, Robin ED. Pulmonary mechanics during pregnancy. $\mathrm{J}$ Clin Invest. 1967 Jun;46(6):945-52.

6. Svensson M, Lindberg E, Naessen T, Janson C. Risk factors associated with snoring in women with special emphasis on body mass index: a populationbased study. Chest. 2006 Apr;129(4):933-41.

7. Karacan I, Heine W, Agnew HW, Williams RL, Webb WB, Ross JJ. Characteristics of sleep patterns during late pregnancy and the postpartum periods. American Journal of Obstetrics and Gynecology. 1968 Jul 1;101(5):579-86. 
8. Suzuki S, Dennerstein L, Greenwood KM, Armstrong SM, Satohisa E. Sleeping patterns during pregnancy in Japanese women. J Psychosom Obstet Gynaecol. 1994 Mar;15(1):19-26.

9. Lao TT, Chin RK, Swaminathan R. Thyroid function in pre-eclampsia. $\mathrm{Br} \mathrm{J}$ ObstetGynaecol. 1988 Sep;95(9):880-3.

10. Sardana D, Nanda S, Kharb S. Thyroid hormones in pregnancy and preeclampsia. J Turk GerGynecol Assoc. 2009 Sep 1;10(3):168-71.

11. Kumar A, Ghosh BK, Murthy NS. Maternal thyroid hormonal status in preeclampsia. Indian J Med Sci. 2005 Feb;59(2):57-63

12. Kumar A, Gupta N, Nath T, Sharma JB, Sharma S. Thyroid function tests in pregnancy. Indian J Med Sci. 2003 Jun;57(6):252-8.

13. Leung AM. Thyroid function in pregnancy. J Trace Elem Med Biol. 2012 Jun;26(0):137-40.

14. Glinoer D. The regulation of thyroid function in pregnancy: pathways of endocrine adaptation from physiology to pathology. Endocr Rev. 1997 Jun;18(3):404-33.

15. Başbuğ M, Aygen E, Tayyar M, Tutuş A, Kaya E, Oktem O. Correlation between maternal thyroid function tests and endothelin in preeclampsiaeclampsia. Obstet Gynecol. 1999 Oct;94(4):551-5.

16. Mandal D, Manda S, Rakshi A, Dey RP, Biswas SC, Banerjee A. Maternal obesity and pregnancy outcome: a prospective analysis. J Assoc Physicians India. 2011 Aug;59:486-9
17. Kazemian E, Sotoudeh G, DorostyMotlagh AR, Eshraghian MR, Bagheri M. Maternal obesity and energy intake as risk factors of pregnancy-induced hypertension among Iranian women. J Health PopulNutr. 2014 Sep;32(3):486- 93. 